\title{
A Review of the Current Status of Follow-Up Techniques to Study Known Extrasolar Planets
}

\author{
David Charbonneau \\ California Institute of Technology, MC 105-24 (Astronomy), \\ 1200 E. California Blvd., Pasadena, CA 91125 USA \\ email:dc@caltech.edu
}

\begin{abstract}
I present a review of observational efforts to study known extrasolar planets by methods that are complementary to the radial velocity technique. I describe the current state of attempts to detect and characterize such planets by astrometry, by reflected light, by thermal emission, by transit photometry, by atmospheric transmission spectroscopy, by planet-induced chromospheric activity, and by long-wavelength radio emission. With a few exceptions, these efforts have yielded only upper limits. Nonetheless, the diversity and vivacity of these pursuits has rapidly pushed many of these techniques into the realm where realistic models of the planets and their atmospheres can now be confronted.
\end{abstract}

\section{Introduction}

Doppler surveys of nearby Sun-like stars continue to supply the bulk of information by which we are learning about the planets that orbit our stellar neighbors. The current state-of-the-art in radial velocity precision did not come easily: It is the result of years of dedicated effort by numerous groups to develop the optimal instrumental methods, prior to the announcement of the first successful detections. By analogy, dozens of research groups are now exploring novel techniques to follow-up and characterize these newly-detected planets. Many of these methods were considered completely unrealistic prior to discovery of the great diversity of planetary systems, when only our own Solar system served as a guide. Notably, several techniques have been fueled by the detection of gas giants that are either significantly more massive, or closer to the central star, than any object in our Solar system. I describe here the current sensitivity of several of these techniques, discussing wherever possible published data, and the corresponding upper limits and/or detections. I provide an extensive reference list so that this paper may also serve as gateway for individuals new to the field: Such studies will likely require many teams and years of development to find the optimal observing strategy.

\section{Astrometry}

Benedict et al. (2002) report the first astrometrically determined mass of an extrasolar planet: Capitalizing on the high-precision astrometry enabled by the 
HST Fine Guidance Sensors, they have detected the astrometric motion of the GJ 876 due to its outer orbiting planet (GJ $876 \mathrm{~b}, P=61.0 \mathrm{~d}$; the inner planet, GJ $876 \mathrm{c}$, has a period of $P=30.1 \mathrm{~d}$ ). Combining the astrometry with the radial velocity measurements, they solve for the perturbation semi-major axis $\alpha=0.25 \pm 0.06$ mas, inclination $i=84^{\circ} \pm 6^{\circ}$, as well as the GJ 876 absolute parallax, $\pi_{\text {bs }}=214.6 \pm 0.2$ mas. Since the system is very close to edge on, the planetary mass they determine, $M_{p}=1.89 \pm 0.34 M_{\mathrm{Jup}}$, is indeed the minimum mass derived from radial velocities alone. The high inclination indicates that the one or both of the planets may indeed lie in a transiting configuration. Furthermore, the dynamical interaction of the two planets causes the orbital elements to vary in time, indicating that one of the planets may enter a transiting configuration, even if this is not currently the case.

By combining Hipparcos astrometry with radial velocity measurements, Han, Black, \& Gatewood (2001) argue that at least 4 of the 30 companions with planetary minimum masses that they examined possess stellar mass $(M>$ $\left.80 M_{\text {Jup }}\right)$, implying that these orbits are seen nearly face-on $\left(i<1^{\circ}\right)$. Pourbaix $\&$ Arenou (2001) present a detailed discussion demonstrating that these conclusions are likely an artifact of the reduction procedure as applied to the Hipparcos data. Astrometry of several additional extrasolar planet systems are presented in detail in the literature: Gatewood, Han, \& Black (2001) claim an inclination of $i=0.5^{\circ}$ and a mass of $M=115 M_{\mathrm{Jup}}$ for the companion to $\rho \mathrm{CrB}$ (which has a minimum mass of $M \sin i=1.04 M_{\mathrm{Jup}}$ ), and Zucker \& Mazeh (2000) find a true mass of $M=38 M_{\text {Jup }}$ for the companion to HD 10697 (which has a minimum mass of $M_{p} \sin i=6.4 M_{\mathrm{Jup}}$ ), indicating a brown dwarf companion rather than a planet. McGrath et al. (2002, 2003) have used HST/FGS to constrain the astrometric motion due to the companion to $\rho^{1}$ Cnc to less than 0.3 mas $(5 \sigma)$, ruling out the preliminary Hipparcos value of 1.15 mas. They derive an upper limit on the companion mass of $30 M_{\mathrm{Jup}}$, confirming that the object is substellar.

Currently, Benedict et al. (2003) are using HST/FGS to monitor the astrometric motion of both $\epsilon$ Eri and $v$ And. They plan to monitor these systems for 3 years, but based on the high precision (1 mas) of the data gathered so far (covering baselines of 2 years and 1 year, respectively), they can already offer interesting upper limits on the companion masses.

\section{Reflected Light: Direct Spectroscopic Separation}

Planets shine in reflected light with a flux (relative to their stars) of

$$
\left(\frac{f_{p}}{f_{*}}\right)_{\lambda}(\alpha)=\left(\frac{R_{p}}{a}\right)^{2} p_{\lambda} \Phi_{\lambda}(\alpha),
$$

where $a$ is the semi-major axis, $p_{\lambda}$ is the geometric albedo, and $\Phi_{\lambda}(\alpha)$ is the phase function (the flux from the planet when viewed at a phase angle $\alpha$ relative to the flux received when the planet is at opposition). The system can thus be pictured as an extreme double-lined spectroscopic binary: The secondary spectrum is exceptionally faint $\left(f_{p} / f_{*} \sim 1 \times 10^{-4}\right)$, but very well separated spectroscopically, as the orbital velocities for these hot Jupiters are typically of order $100 \mathrm{~km} \mathrm{~s}^{-1}$, 
much greater than the typical stellar line widths of $2-10 \mathrm{~km} \mathrm{~s}^{-1}$. The challenge is to detect the secondary spectrum, and thus derive the planetary mass (from the projected velocity separation of the planet and star), as well as estimates of the geometric albedo and planetary radius.

Charbonneau et al. (1999) placed the first significant upper limit on the reflected light from an extrasolar planet. They observed $\tau$ Boo (orbital period $P=3.31 \mathrm{~d}, M_{p} \sin i=4.38 M_{\mathrm{Jup}}$ ) with Keck/HIRES for 3 nights under intermittent clouds. Assuming a planetary radius of $R_{p}=1.2 R_{\mathrm{Jup}}$, they placed an upper limit on the average planetary geometric albedo of $p<0.3$ over the wavelength range $465-499 \mathrm{~nm}$. This limit is valid for high orbital inclinations $\left(i>70^{\circ}\right)$, and a less restrictive limit applies for lower inclinations. Recently, Leigh et al. (2003b) have presented a combined analysis of their WHT/UES data of the $\tau$ Boo system from 1998, 1999, \& 2000. Assuming a planetary radius of $R_{p}=1.2 R_{\mathrm{Jup}}$, an orbital inclination of $i=36^{\circ}$, and a Venus-like phase function, they present an upper limit on the average geometric albedo (taken over their wavelength range of $385-611 \mathrm{~nm}$ ) of $p<0.39$ (for a conservative false alarm probability of $0.1 \%$ ). This system is distinctive in that there is independent evidence that the star is tidally locked at the $3.31 \mathrm{~d}$ orbital period (Baliunas et al. 1997). As a result, the reflected light spectrum is likely non-rotationally broadened (with a line width determined by the macroturbulent velocity, $v_{\text {turb }} \simeq 4 \mathrm{~km} \mathrm{~s}^{-1}$ ), which should facilitate its separation from the rotationally-broadened stellar features $\left(v_{*} \sin i \simeq 15 \mathrm{~km} \mathrm{~s}^{-1}\right)$.

Similar studies have been carried out for two other hot Jupiter systems: Based on 3 nights of WHT/UES spectra, Collier Cameron et al. (2002) derived an upper limit for the geometric albedo of $v$ And $\mathrm{b}\left(P=4.62 \mathrm{~d}, M_{p} \sin i=\right.$ $\left.0.73 M_{\mathrm{Jup}}\right)$ of $p<0.98\left(R_{p} / R_{\mathrm{Jup}}\right)^{-2}(0.1 \%$ false alarm probability $)$. They consider two model spectra from Sudarsky et al. (2000), and place limits on the planetary radius based on these models. The first is a Class V "Roaster" model atmosphere that includes the effects of stellar insolation. This model yields a predicted geometric albedo of $p=0.42$ when averaged over their band pass, and thus requires that $R_{p}<1.51 R_{\text {Jup }}$. The second model they consider is an isolated Class IV model atmosphere, which predicts a lower geometric albedo of $p=0.19$, and yields a correspondingly weaker limit on the planetary radius of $R_{p}<2.23 R_{\text {Jup. }}$. Using 4 nights of VLT/UVES data, Leigh et al. (2003c) have presented a similar study of the hot Jupiter system HD $75289(P=3.51 \mathrm{~d}$, $\left.M_{p} \sin i=0.46 M_{\mathrm{Jup}}\right)$. Assuming an orbital inclination of $i=60^{\circ}$ and a planetary radius of $R_{p}=1.6 R_{\mathrm{Jup}}$, they placed an upper limit on the average geometric albedo of $p<0.12$, with a false alarm probability of $0.1 \%$.

Charbonneau \& Noyes (2000) and, more recently, Leigh et al. (2003a) have discussed future prospects for reflected-light studies. Two attractive targets for which no such studies have been published to date are 51 Peg $(P=4.23 \mathrm{~d}$, $\left.M_{p} \sin i=0.48 M_{\mathrm{Jup}}\right)$ and HD $179949\left(P=3.09 \mathrm{~d}, M_{p} \sin i=0.84 M_{\mathrm{Jup}}\right)$. The HD 209458 system $\left(P=3.52 \mathrm{~d}, M_{p} \sin i=0.69 M_{\text {Jup }}\right)$ would require longer integration times due to its faintness $(V=7.6)$, yet the interpretation of such data would be greatly simplified: Since the orbital inclination $\left(i=86.7^{\circ}\right)$ and planetary radius $\left(R_{p}=1.35 R_{\mathrm{Jup}}\right)$ are known, the geometric albedo can be estimated unambiguously from the flux ratio. 


\section{Reflected Light: Photometric Monitoring}

A more direct method to study the albedo and scattered light curves of hot Jupiters is ultra-high precision photometry capable of measuring the photometric modulation that results from the varying illumination of the planet as it orbits the central star. Ground-based efforts to detect the reflected-light modulation from extrasolar planets are typically limited by atmospheric extinction effects to a precision of $0.1 \%$ (see Kenworthy \& Hinz 2003 for a description of such an effort). Thus it appears that the required precision will be available only from space for the time being. The MOST satellite (Matthews et al. 2000; Walker et al. 2003) was launched 30 June 2003, and possesses just such a capability: Although its primary science goal is the detection and characterization of rapid acoustic oscillations in Sun-like stars $(\nu \simeq 0.5-6 \mathrm{mHz})$, it should have the precision to detect the variation caused by several known hot Jupiters $(\nu \simeq 0.003 \mathrm{mHz})$. MOST conducts photometry in a single bandpass $(350-700 \mathrm{~nm})$ and thus will provide no spectral information. Nonetheless, the derived phase variation should be diagnostic of the principal sources of scattering in the planetary atmosphere. Three known hot-Jupiter systems, $51 \mathrm{Peg}, \tau$ Boo, and HD 209458, are on the MOST primary target list. Recently, Green et al. (2003) have presented a detailed analysis of the ability of MOST to measure such variations. Assuming a conservative detection threshold of $4.2 \mu \mathrm{mag}(3 \sigma)$, they find that MOST should indeed be able to detect $\tau$ Boo b in reflected light, or place severe constraints on the models of the planet atmosphere.

Jenkins \& Doyle (2003) present a detailed analysis of the ability of the upcoming Kepler Mission to detect previously unknown hot Jupiters by their photometric variation. They find that, depending on the albedo and scattering function, Kepler should reveal $100-760$ new hot Jupiters (with orbital periods less than 7 days) by their reflected light, assuming a conservative limit of not more than one false positive rate over the Kepler campaign. The primary detectability determinant (other than the model of the planetary atmosphere) is the stellar rotation period, although stellar brightness is significant for $R>12.5$. The upcoming CNES COROT Mission (Schneider et al. 1998) may also detect hot Jupiters by this effect.

\section{Transit Photometry}

I do not discuss here the numerous efforts to detect unknown extrasolar planets by the transit method (for a review, see Horne 2003 and Charbonneau 2003a). Rather, I restrict my attention to the two systems that are known to transit.

Numerous groups (Charbonneau et al. 2000; Henry et al. 2000; Jha et al. 2000; Deeg, Garrido, \& Claret 2001) have presented ground-based photometry of the planetary transit of HD 209458 with a typical precision of 2 mmag and a cadence of roughly 10 minutes. At this level of measurement, estimation of the planet radius is frustrated by a significant degeneracy, as it is possible to fit the data by a family of models wherein the ratio $R_{p} / R_{*}$ is preserved. The typical resulting uncertainty in the planetary radius as derived from these ground-based data sets is $10 \%$. In contrast to this situation, Brown et al. (2001) observed four transits with HST/STIS, and achieved a precision of $0.1 \mathrm{mmag}$ 
and a rapid cadence of $80 \mathrm{~s}$. These data constrained the slope and duration of ingress and egress with sufficient quality to break the above degeneracy. The resulting estimates of the planetary and stellar radii were $R_{p}=1.35 \pm 0.06 R_{\mathrm{Jup}}$ and $R_{*}=1.15 \pm 0.05 R_{\odot}$. Combining the planetary radius with the planetary mass $M_{p}=0.69 \pm 0.05 M_{\text {Jup }}$, Brown et al. (2001) deduce an average density of $\rho=0.35 \mathrm{~g} \mathrm{~cm}^{-3}$, a surface gravity of $g=943 \mathrm{~cm} \mathrm{~s}^{-2}$, and an escape velocity of $v_{e}=43 \mathrm{~km} \mathrm{~s}^{-1}$. They also ruled out the presence of planetary satellites larger than $1.2 R_{\oplus}$ (based on photometric residuals) or more massive than $3 R_{\oplus}$ (based on timing residuals), and placed constraints on the presence of opaque circumplanetary rings. Schultz et al. (2003) observed several transits with the HST Fine Guidance Sensors, which yielded an ultra-rapid cadence of $0.025 \mathrm{~s}$, and a typical signal-to-noise ratio of 80 . These observations target times of ingress and egress, and can be used to search for timing offsets indicative of planetary satellites. Furthermore, the combination of the Brown et al. (2001) and Schultz et al. (2003) data sets, each of which permit an accurate determination of the time of center of transit, yields an extremely precise value of the orbital period. This should effectively remove any timing uncertainties in the planning and interpretation of future data sets.

OGLE-TR-56 $\mathrm{b}$ is the first extrasolar planet detected by the transit method (Udalski et al. 2002a,b; Konacki et al. 2003a). The period deduced from recent Doppler observations of the system (Torres et al. 2003) confirms that found from the photometric data, and the amplitude of the Doppler shift demonstrates that the companion is indeed of planetary mass. The precision of the radial velocity observations is relatively coarse (typically $100 \mathrm{~m} \mathrm{~s}^{-1}$, due to the much fainter magnitude of the star than those typically surveyed). The best estimates of the planetary radius, $R_{p}=1.23 \pm 0.16 R_{\mathrm{Jup}}$, and mass, $M_{p}=1.45 \pm 0.23 M_{\mathrm{Jup}}$, imply a much greater density $\rho=1.0 \mathrm{~g} \mathrm{~cm}^{-3}$ than that of HD $209458 \mathrm{~b}$. The semimajor axis of OGLE-TR-56 b (0.0225 AU) is less than half that of HD $209458 \mathrm{~b}$ $(0.0468 \mathrm{AU})$, and thus comparison of these objects may offer us insight into the importance of insolation upon the planetary radius. Several other targets from the OGLE campaign remain under scrutiny (Konacki et al. 2003b).

\section{Transmission Spectroscopy of the Atmosphere and Exosphere}

Charbonneau et al. (2002) observed four transits of HD 209458 with HST/STIS, and detected an increase in transit depth of $(2.32 \pm 0.57) \times 10^{-4}$ in a narrow bandpass centered on the sodium resonance lines near $589.3 \mathrm{~nm}$. They rule out alternate explanations of this dimming (such as stellar limb darkening), and conclude that the effect is due to absorption in the planetary atmosphere. The signal amplitude is roughly $1 / 3$ that predicted from model atmospheres that are cloudless and contain a solar abundance of sodium in atomic form. A possible explanation for this decrement is the presence of a high-altitude cloud deck (with cloud tops above 0.4 mbar). An alternative reason is the depletion of atomic sodium, either as a result of chemical reaction into molecules such as $\mathrm{NaCl}$ and $\mathrm{Na}_{2} \mathrm{~S}$, or indicative of a global metal abundance that is significantly reduced relative to solar.

Similarly, Vidal-Madjar et al. (2003) observed three transits of HD 209458 with HST/STIS, but in a bandpass centered on the Ly $\alpha$ feature (notably, these 
UV wavelengths use the STIS MAMA detectors, as opposed to the STIS CCD device). They found a transit depth of $15 \pm 4 \%$, corresponding to an equivalent size of $4.3 R_{\mathrm{Jup}}$. As this is in excess of the Roche limit of $3.6 R_{\mathrm{Jup}}$, they conclude that some hydrogen atoms must be escaping from the planet (there is also some independent spectral evidence that the atoms have a large velocity relative to the planet). Although the minimum escape rate required by the data would reduce the planetary mass by only a negligible amount $(0.1 \%)$ when integrated over the age of the system, significantly higher mass-loss rates are also permitted.

There have been several ground-based efforts to detect additional atmospheric features in absorption, all of which have been frustrated by variability in the telluric spectrum and the spectrograph. Moutou et al. (2001) observed a portion of a planetary transit with VLT/UVES, spanning the wavelength range $328-669 \mathrm{~nm}$, and performed a general search for prominent absorption features. Their precision was typically $1 \%$ (worse at shorter wavelengths), and thus they were restricted to looking for exospheric (as opposed to atmospheric) features. Such an exosphere is undoubtedly the site of complex photochemistry, which results in a long list of potential atomic and molecular species (and their associated ions). Moutou et al. (2003a) observed a planet transit with the VLT/ISAAC instrument to search for the He I line at $1083 \mathrm{~nm}$. They present an upper limit of $0.5 \%(3 \sigma)$ for a $0.3 \mathrm{~nm}$ band centered on the feature, slightly above the predicted signal strength of $0.25 \%$. They outline improvements that would likely allow similar observations to achieve a detection limit of $0.1 \%$. Iro et al. (2003) $\&$ Moutou et al. (2003b) have also presented preliminary results from associated efforts.

Brown, Libbrecht \& Charbonneau (2002) present Keck/NIRSPEC data from a preliminary attempt to detect features from the $\mathrm{CO}$ molecule at $2.3 \mu \mathrm{m}$. The $\mathrm{CO}$ molecule is diagnostic of the temperature in the planetary atmosphere, as the equilibrium temperature of $\mathrm{HD} 209458 \mathrm{~b}(\sim 1400 \mathrm{~K})$ falls in the regime where carbon migrates from $\mathrm{CO}$ to $\mathrm{CH}_{4}$ (with the latter preferred at lower temperatures). The observing conditions, however, were not ideal: The transit was not visible in its entirety from their longitude, and the weather was poor. They present an upper limit that is a factor of 3 above reasonable models of the planetary atmosphere, and argue that future attempts should be able to achieve the precision required to test directly models of the planetary atmosphere. Harrington et al. (2003) discuss a large effort to detect IR transmission features with data gathered with Palomar, Keck, VLT, and IRTF.

Rauer et al. (2000) present spectra of $51 \mathrm{Peg}$ gathered with the SWS instrument on ISO. Their sensitivity is sufficient to place limits on the presence of features from an extended exosphere. However, the lack of constraints on the orbital inclination prevent them from directly constraining models of the planetary exosphere.

\section{Infrared Emission}

Infrared wavelengths offer a far more favorable contrast ratio between the planet and star than visible light. As a result, there have been numerous efforts to detect directly IR spectroscopic features from the planetary atmosphere. Lucas \& Roche (2002) examined five systems (HD 187123, $51 \mathrm{Peg}, \tau$ Boo, $v$ And, and 
HD 209458) with the UKIRT Cooled Grating Spectrograph CGS4 at $K$ and $L^{\prime}$. They searched for the spectroscopic edges arising from $\mathrm{H}_{2} \mathrm{O}$ and $\mathrm{CH}_{4}$, neither of which are expected to be present in the stellar spectrum. Their typical $3 \sigma$ upper limits for water features were at the level of one part in several hundred. The corresponding limits on the planetary radius ranged from $2.6-5 R_{\text {Jup }}$ (depending upon the individual object and atmospheric model) and hence were insufficient to test directly realistic models of the planetary radii and atmospheres. Nonetheless, they note that similar studies with SIRTF would likely attain the required precision. Wiedemann, Deming, \& Bjoraker (2001) present the results of a sensitive search for methane in the infrared spectrum of $\tau$ Boo, using the IRTF CHSELL spectrometer. In contrast to Lucas \& Roche (2002), they explicitly search for a signal that varies according to the known planetary orbit. They tentatively claim a detection of methane features in the planetary atmosphere (the signal strength is $2 \times 10^{-4}$ of the stellar continuum), but they caution that the significance of the detection is marginal $(2.4 \sigma)$.

In a couple of recent papers, Richardson et al. (2003a,b) have presented strong limits on the infrared spectrum of HD 209458 b. Their method of "occultation spectroscopy" capitalizes on the transiting geometry to search for the disappearance and reappearance of spectral features at the times of ingress and egress. They argue that the signal amplitude available to this method exceeds the analogous signal from transmission spectroscopy for wavelengths longward of approximately $2.6 \mu \mathrm{m}$. In Richardson et al. (2003a), they present the analysis of VLT/ISAAC spectra with a resolution of $R=3300$ gathered during two times of secondary eclipse. They search for planetary methane features near $3.6 \mu \mathrm{m}$, and are able to rule out irradiated, low-opacity (cloudless), low-albedo, thermochemical-equilibrium models of the planetary atmosphere. They are not, however, sensitive to all reasonable models of the planetary atmosphere: In particular, cloudy models (as favored by the small amplitude of the sodium feature detected by transmission spectroscopy; Charbonneau et al. 2002) would yield substantially weaker features which would be below their detection threshold. Richardson et al. (2003b) present data spanning the $1.9-4.2 \mu \mathrm{m}$ region gathered with the IRTF/SpeX instrument with a resolution of $R=1500$. They give upper limits on a continuum peak near $2.2 \mu \mathrm{m}$ that would result from $\mathrm{CO}$ and $\mathrm{H}_{2} \mathrm{O}$ features in the planetary atmosphere. These upper limits are at the level of roughly $3 \times 10^{-4}$ of the stellar flux, a factor of two below some models of the planetary atmosphere. They are able to rule out cloudless models in which there is a strong day-night temperature asymmetry, as such models yield a steep temperature-pressure profile to which their experiment is most sensitive.

The secondary eclipse need not occur exactly half a period after the primary eclipse. The current upper limit on the orbital eccentricity, $e=0.00967 \pm 0.014$ (G. Marcy, personal communication) is indeed consistent with zero (as expected from tidal circularization), but could conceivably be as high as $\sim 0.04$. An eccentricity of $e \simeq 0.04$ could shift the time of secondary eclipse by as much as 2 hours (depending on the longitude of periastron $\omega$; see Charbonneau 2003b for details). The detection of a significant offset would place a lower limit on the eccentricity (and the radial velocity measurements provide an upper bound). Conversely, upper limits on the offset would provide even more stringent constraints upon the eccentricity, and thus bear upon the mechanisms that compete to pump and damp the orbital eccentricity. Thus, experiments that attempt to 
detect the secondary eclipse must account for the additional complication that the event may shifted by as much as 2 hours, but this possibility also provides us with a additional tool with which to study the planet and its orbit.

\section{Planet-Induced Chromospheric Activity}

Cuntz, Saar \& Musielak (2000) investigate several possible interactions between extrasolar planets and their parent stars that may result in stellar chromospheric and coronal activity enhancement. These effects will be due predominantly to a combination of tidal and magnetic effects. The origin of any observed activity could be distinguished observationally, since tidal effects should reveal a periodicity of $P / 2$ (where $P$ is the planetary orbital period), whereas magnetic effects should follow a period of $P$. Both tidal and magnetic effects would decrease rapidly in intensity with semi-major axis $a$ (with dependencies of $\propto a^{-3}$ and $\propto a^{-2}$, respectively). Thus the best targets are the hot Jupiter systems. These planets may, however, have magnetic fields that are significantly weaker than that of Jupiter, since they are likely tidally locked, and thus their rotation periods are typically 10 times greater than the Jovian value.

Saar \& Cuntz (2001) searched for periodicities in the Ca II infrared triplet, indicative of chromospheric activity, in 7 extrasolar planet systems $(\tau$ Boo, $51 \mathrm{Peg}, v$ And, $\rho^{1} \mathrm{Cnc}, \rho \mathrm{CrB}, 70 \mathrm{Vir}$, and GJ 876). They examined extant Lick spectra, with a typical resolution $R=50,000$ and a signal-to-noise ratio of 200 , which were gathered as part of the extrasolar planet search program. For each spectrum, they evaluate the index $S_{\mathrm{IR}}=\left\langle F_{\mathrm{em}}\right\rangle / F_{\text {con }}^{*}$, where $\left\langle F_{\mathrm{em}}\right\rangle$ is the emission flux centered on the core of the $866.2 \mathrm{~nm}$ line, and $F_{\text {con }}^{*}$ is the median continuum flux in a line-free region on either side of the line. They found no significant signal at either period $(P / 2$ or $P)$, with upper limits of $3-10 \%$, although for several stars they saw low-statistical-significance indications of the stellar rotational signature.

Shkolnik, Walker, \& Bohlender (2003a) have recently detected the enhancement of $\mathrm{Ca}$ II $\mathrm{H}$ and $\mathrm{K}$ emission in phase with the orbit $(P=3.09 \mathrm{~d})$ of the hot Jupiter companion to HD 179949, indicating a magnetic (rather than tidal) origin of the signal. This detection may offer the community's first access to the magnetosphere of an extrasolar planet. For this effort, they gathered multiepoch high-resolution spectra $(R=110,000)$ with a typical signal-to-noise of 500 (in the continuum adjacent to the Ca II $\mathrm{H}$ and $\mathrm{K}$ lines). They also monitored a strong photospheric $\mathrm{Al}$ I line, which revealed no changes, indicating that the periodic variation did indeed originate in the chromosphere. As of yet, there is no independent evidence that the stellar rotation period is the same as the orbital period. Shkolnik et al. (2003b) are monitoring 4 other hot Jupiter systems. Three of these ( $\tau$ Boo, $v$ And, and HD 209458) have revealed significant nightly variations, but, as of yet, the variations do not conclusively show phase coherence with the planetary orbits. The group is monitoring $51 \mathrm{Peg}$, but has seen no clear evidence for variability. 


\section{Radio Emission}

Even before the Doppler detections of numerous extrasolar planets, searches for long-wavelength radio emission from planets orbiting neighboring stars had been carried out (Wingleee, Dulk \& Bastian 1986). Such searches are motivated by the observation that the polar regions of all of the Solar system gas giants are the sites of intense auroral-related radio emission. In particular, the coherent cyclotron radiation from Jupiter often exceeds $10^{10} \mathrm{~W}$. It is variable in time by a factor of 1000 , and the emission is correlated exponentially with the input velocity and power of the solar wind. Several scaling laws have been proposed to relate the approximate radio power for the planets of the solar system. These "Radiometric Bode's Laws" suggest that the radiated power scales directly with the planetary mass, and inversely with the three-halves power of the semi-major axis, $\propto M_{p} a^{-3 / 2}$. Since we now know of several nearby Sun-like stars that are host to Jupiter-mass planets at a distance of $0.05 \mathrm{AU}$, the scaling laws from the Solar system suggest that the radio emission from these objects could exceed Jupiter by a factor of 1000 (see discussion in Farrell et al. 2003 for more details). Farrell et al. (1999), Bastian et al. (2000), and Zarka et al. (2001) discuss the emission mechanisms in detail, and make predictions of emitted radio power for several of the known extrasolar planets.

The detection of cyclotron radio emission from extrasolar planets would be a valuable tool (e.g. Bastian et al. 2000): It would permit the characterization of the planetary magnetic field, and the upper limit on the emission frequency would yield the maximum strength of the magnetic field. The sense of circular polarization might indicate from which pole the emission originated, and the elliptical polarization might yield a limit on the plasma density in the planetary magnetosphere. Furthermore, the periodicity of the radio emission would give a direct determination of the rotational period (indeed, the rotation periods for the solar system gas giants are determined by this method, not by the observation of atmospheric features, which rotate differentially). Additional periodicities in the emission might indicate the presence of planetary satellites, again by analogy with the planets of the Solar system.

Farrell et al. (2003) identified the hot Jupiter system $\tau$ Boo $\left(M_{p}=\right.$ $4.38 \mathrm{M}_{\mathrm{Jup}}$ ) as the optimal target, as the scaling favors massive planets at small orbital separations. At a distance of $10 \mathrm{pc}$, the radio power from Jupiter would be approximately $0.023 \mathrm{mJy}$. Based on the scaling law, $\tau$ Boo b could yield

$$
\begin{aligned}
S_{p} & \simeq\left(\frac{M_{p}}{M_{\mathrm{Jup}}}\right)\left(\frac{a_{p}}{a_{\mathrm{Jup}}}\right)^{-3 / 2}\left(\frac{d}{10 \mathrm{pc}}\right)^{-2} S_{\mathrm{Jup}, 10 \mathrm{pc}} \\
& \simeq 4.38(0.046 / 5.2)^{-3 / 2}(15.6 / 10)^{-2} 0.023 \mathrm{mJy} \\
& \simeq 50 \mathrm{mJy} .
\end{aligned}
$$

Observing at the Very Large Array (VLA) in May 2002, they placed an upper limit of $120 \mathrm{mJy}$ for such emission at $74 \mathrm{MHz}$. Although this limit does not yet reach the predicted signal level, it demonstrates that such searches are nearly within reach of current instrumentation, and should be accessible to the nextgeneration arrays. Furthermore, such emission is likely quite variable in time. 
Thus it could be that the emission from $\tau$ Boo b does occasionally exceed the current capabilities of the VLA.

Bastian et al. (2000) have conducted VLA observations of seven extrasolar planets (51 Peg, $v$ And, $\rho^{1}$ Cnc, $47 \mathrm{UMa}, \tau$ Boo, $70 \mathrm{Vir}$, and HD 114762) at $333 \mathrm{MHz}$ and $1465 \mathrm{MHz}$, where the sensitivities are greater, but the predicted signals are much weaker. Their typical $1-\sigma$ sensitivities were $1-10 \mathrm{mJy}$ at $333 \mathrm{MHz}$, and $0.02-0.07 \mathrm{mJy}$ at $1465 \mathrm{MHz}$. They state upper limits for each system. They also observed the $47 \mathrm{UMa}$ system $\left(M_{p} \sin i=2.54 M_{\mathrm{Jup}}\right.$, $P=1089 \mathrm{~d}$ ), at $74 \mathrm{MHz}$ with a sensitivity of $50 \mathrm{mJy}$, but did not detect a signal. A weaker signal is expected for $47 \mathrm{UMa}$ than for the hot Jupiter system $\tau$ Boo, due to the much larger semi-major axis.

\section{References}

Baliunas, S. L., Henry, G. W., Donahue, R. A., Fekel, F. C., \& Soon, W. H. 1997, ApJ, 474, L119

Bastian, T. S., Dulk, G. A., \& Leblanc, Y. 2000, ApJ, 545, 1058

Benedict, G. F. et al. 2003, AAS/Division for Planetary Sciences Meeting, 35, 7.10

Benedict, G. F. et al. 2002, ApJ, 581, L115

Brown, T. M., Charbonneau, D., Gilliland, R. L., Noyes, R. W., \& Burrows, A. 2001, ApJ, 552, 699

Brown, T. M., Libbrecht, K. G., \& Charbonneau, D. 2002, PASP, 114, 826

Charbonneau, D. 2003a, in Space Science Reviews: ISSI Workshop on Planetary Systems and Planets in Systems, ed. S. Udry, W. Benz, \& R. von Steiger (Dordrecht: Kluwer), astro-ph/0302216

Charbonneau, D. 2003b, ASP Conf. Ser. 294: Scientific Frontiers in Research on Extrasolar Planets, ed. D. Deming \& S. Seager (San Francisco: ASP), 449, astro-ph/0209517

Charbonneau, D., Brown, T. M., Latham, D. W., \& Mayor, M. 2000, ApJ, 529, L45

Charbonneau, D., Brown, T. M., Noyes, R. W., \& Gilliland, R. L. 2002, ApJ, 568,377

Charbonneau, D. \& Noyes, R. W. 2000, ASP Conf. Ser. 219: Disks, Planetesimals, and Planets, eds. F. Garzon \& T. J. Mahoney (San Francisco: ASP), 461, astro-ph/0002489

Charbonneau, D., Noyes, R. W., Korzennik, S. G., Nisenson, P., Jha, S., Vogt, S. S., \& Kibrick, R. I. 1999, ApJ, 522, L145

Collier Cameron, A., Horne, K., Penny, A., \& Leigh, C. 2002, MNRAS, 330, 187

Cuntz, M., Saar, S. H., \& Musielak, Z. E. 2000, ApJ, 533, L151

Deeg, H. J., Garrido, R., \& Claret, A. 2001, New Astronomy, 6, 51

Farrell, W. M., Desch, M. D., Lazio, T. J., Bastian, T., \& Zarka, P. 2003, ASP Conf. Ser. 294: Scientific Frontiers in Research on Extrasolar Planets, ed. D. Deming \& S. Seager (San Francisco: ASP), 151

Farrell, W. M., Desch, M. D., \& Zarka, P. 1999, JGR, 104, 14025 
Gatewood, G., Han, I., \& Black, D. C. 2001, ApJ, 548, L61

Green, D., Matthews, J., Seager, S., \& Kuschnig, R. 2003, ApJ, 597, 590

Han, I., Black, D. C., \& Gatewood, G. 2001, ApJ, 548, L57

Harrington, J., Deming, D., Goukenleuque, C., Matthews, K., Richardson, L. J.,

Steyert, D., Wiedemann, G., \& Zeehandelaar, D. 2003, ASP Conf. Ser. 294:

Scientific Frontiers in Research on Extrasolar Planets, ed. D. Deming \&

S. Seager (San Francisco: ASP), 471

Henry, G. W., Marcy, G. W., Butler, R. P., \& Vogt, S. S. 2000, ApJ, 529, L41

Horne, K. 2003, ASP Conf. Ser. 294: Scientific Frontiers in Research on Extrasolar Planets, ed. D. Deming \& S. Seager (San Francisco: ASP), 361, astro-ph/0301249

Iro, N., Coustenis, A., Moutou, C., Lajous, N., Mayor, M., \& Queloz, D. 2003, ASP Conf. Ser.: XIXth IAP colloquium: Extrasolar Planets: Today and Tomorrow, astro-ph/0311073

Jenkins, J. M. \& Doyle, L. R. 2003, ApJ, 595, 429

Jha, S., Charbonneau, D., Garnavich, P. M., Sullivan, D. J., Sullivan, T., Brown, T. M., \& Tonry, J. L. 2000, ApJ, 540, L45

Kenworthy, M. A. \& Hinz, P. M. 2003, PASP, 115, 322

Konacki, M., Torres, G., Jha, S., \& Sasselov, D. D. 2003a, Nature, 421, 507

Konacki, M., Torres, G., Sasselov, D. D., \& Jha, S. 2003b, ApJ, 597, 1076

Leigh, C., Collier Cameron, A., \& Guillot, T. 2003a, MNRAS, in press, astro$\mathrm{ph} / 0308414$

Leigh, C., Collier Cameron, A., Horne, K., Penny, A., \& James, D. 2003b, MNRAS, 344, 1271

Leigh, C., Collier Cameron, A., Udry, S., Donati, J., Horne, K., James, D., \& Penny, A. 2003c, MNRAS, 346, L16

Lucas, P. W. \& Roche, P. F. 2002, MNRAS, 336, 637

Matthews, J. M. et al. 2000, ASP Conf. Ser. 203: IAU Colloq. 176: The Impact of Large-Scale Surveys on Pulsating Star Research, ed. L. Szabados \& D. Kurtz (San Francisco: ASP), 74

McGrath, M. A. et al. 2003, ASP Conf. Ser. 294: Scientific Frontiers in Research on Extrasolar Planets, ed. D. Deming \& S. Seager (San Francisco: ASP), 145

McGrath, M. A. et al. 2002, ApJ, 564, L27

Moutou, C., Coustenis, A., Iro, N., Schneider, J., Mayor, M., \& Queloz, D. 2003a, ASP Conf. Ser. 294: Scientific Frontiers in Research on Extrasolar Planets, ed. D. Deming \& S. Seager (San Francisco: ASP), 475

Moutou, C., Coustenis, A., Schneider, J., Queloz, D., \& Mayor, M. 2003b, A\&A, 405,341

Moutou, C., Coustenis, A., Schneider, J., St Gilles, R., Mayor, M., Queloz, D., \& Kaufer, A. 2001, A\&A, 371, 260

Pourbaix, D. \& Arenou, F. 2001, A\&A, 372, 935

Rauer, H., Bockelée-Morvan, D., Coustenis, A., Guillot, T., \& Schneider, J. 2000, A\&A, 355, 573 
Richardson, L. J., Deming, D., \& Seager, S. 2003a, ApJ, 597, 581

Richardson, L. J., Deming, D., Wiedemann, G., Goukenleuque, C., Steyert, D., Harrington, J., \& Esposito, L. W. 2003b, ApJ, 584, 1053

Saar, S. H. \& Cuntz, M. 2001, MNRAS, 325, 55

Schneider, J. \& et al. 1998, ASP Conf. Ser. 148: Origins, ed. C. E. Woodward, J. M. Shull, \& H. A. Thronson, Jr. (San Francisco: ASP), 298

Schultz, A. B. et al. 2003, ASP Conf. Ser. 294: Scientific Frontiers in Research on Extrasolar Planets, ed. D. Deming \& S. Seager (San Francisco: ASP), 479

Shkolnik, E., Walker, G. A. H., \& Bohlender, D. A. 2003a, ApJ, 597, 1092

Shkolnik, E., Walker, G. A. H., \& Bohlender, D. A. 2003b, ASP Conf. Ser. 294: Scientific Frontiers in Research on Extrasolar Planets, ed. D. Deming \& S. Seager (San Francisco: ASP), 157

Sudarsky, D., Burrows, A., \& Pinto, P. 2000, ApJ, 538, 885

Torres, G., Konacki, M., Sasselov, D. D., \& Jha, S. 2003, ApJ, submitted, astro$\mathrm{ph} / 0310114$

Udalski, A. et al. 2002a, Acta Astronomica, 52, 1

Udalski, A., Zebrun, K., Szymanski, M., Kubiak, M., Soszynski, I., Szewczyk, O., Wyrzykowski, L., \& Pietrzynski, G. 2002b, Acta Astronomica, 52, 115

Vidal-Madjar, A., Lecavelier des Etangs, A., Désert, J.-M., Ballester, G. E., Ferlet, R., Hébrard, G., \& Mayor, M. 2003, Nature, 422, 143

Walker, G. A. H., et al. 2003, PASP, submitted

Wiedemann, G., Deming, D., \& Bjoraker, G. 2001, ApJ, 546, 1068

Winglee, R. M., Dulk, G. A., \& Bastian, T. S. 1986, ApJ, 309, L59

Zarka, P., Treumann, R. A., Ryabov, B. P., \& Ryabov, V. B. 2001, Ap\&SS, 277, 293 